\title{
IMPLEMENTAÇÃO DE NÚCLEO DE SEGURANÇA DO PACIENTE EM UNIDADE DE PRONTO ATENDIMENTO: PERSPECTIVAS DOS ENFERMEIROS
}

\author{
IMPLEMENTATION OF A PATIENT SAFETY CENTER IN \\ AN EMERGENCY CARE UNIT: NURSES' PERSPECTIVE
}

\section{IMPLEMENTACIÓN DE UN NÚCLEO DE SEGURIDAD PARA EL PACIENTE EN UNA UNIDAD DE ATENCIÓN DE URGENCIA: PERSPECTIVAS DE LAS ENFERMERAS}

\author{
Simone Graziele Silva Cunha ${ }^{1}$ \\ Gabriela Santos Clemence ${ }^{2}$ \\ Larissa Franciele Simões Almeida ${ }^{3}$ \\ Andréia Guerra Siman ${ }^{4}$ \\ Maria José Menezes Brito
}

Como citar este artigo: Cunha SGS, Clemence GS, Almeida LFS, Siman AG, Brito MJM. Implementação de Núcleo de Segurança do Paciente em Unidade de Pronto Atendimento: perspectivas dos enfermeiros. Rev baiana enferm. 2020;34:e36216.

\begin{abstract}
Objetivo: compreender a implementação do Núcleo de Segurança do Paciente em uma Unidade de Pronto Atendimento na perspectiva dos enfermeiros. Método: estudo de caso qualitativo, realizado em uma Unidade de Pronto Atendimento em um município da Região Centro-Oeste de Minas Gerais, Brasil, com a participação de 12 enfermeiros. Os dados foram coletados de setembro a novembro de 2019, por meio de entrevista guiada por roteiro semiestruturado, Técnica do Gibi e observação. Para a análise adotou-se a análise de conteúdo. Resultados: emergiram duas categorias temáticas: Desafios da implementação do Núcleo de Segurança do Paciente e Facilidades da implementação do Núcleo de Segurança do Paciente. Conclusão: foram identificados fragilidades e desafios que impactam no processo de implementação do núcleo e consequentemente no cuidado ao paciente, o que pode servir para subsidiar a implantação de novos Núcleos de Segurança do Paciente em serviços de saúde.
\end{abstract}

Descritores: Serviços Médicos de Emergência. Enfermagem. Segurança do Paciente.

Objective: to comprehend the implementation of the Patient Safety Center in an Emergency Care Unit from the nurses' perspective. Method: qualitative case study, conducted in an Emergency Care Unit in a city in the Midwest Region of Minas Gerais, Brazil, with the participation of 12 nurses. The data were collected from September to November 2019 through an interview guided by a semi-structured script, Comic Book Technique and observation. For the analysis the content analysis was adopted. Results: two thematic categories emerged: Challenges of the implementation of the Patient Safety Center and Facilities of the implementation of the Patient Safety Center. Conclusion: fragilities and

\footnotetext{
Enfermeira. Mestre em Enfermagem. Universidade Federal de Minas Gerais. Belo Horizonte, Minas Gerais, Brasil. simonegscunha@gmail.com. https://orcid. org/0000-0002-0445-4822.

Estudante de Enfermagem. Universidade do Estado de Minas Gerais. Divinópolis, Minas Gerais, Brasil. https://orcid.org/0000-0002-6387- I079.

Estudante de Enfermagem. Universidade do Estado de Minas Gerais. Divinópolis, Minas Gerais, Brasil. https://orcid.org/0000-0002-9667-2025.

Enfermeira. Doutora em Enfermagem. Professora Adjunta da Universidade Federal de Viçosa. Viçosa, Minas Gerais, Brasil. https://orcid.org/0000-000 I-7990-9273.

Enfermeira. Doutora em Administração. Professora Associada da Universidade Federal de Minas Gerais. Belo Horizonte, Minas Gerais, Brasil. https://orcid.org/0000000 I-9183-1982.
} 
challenges were identified that impact on the implementation process of the center and consequently on patient care, which may serve to subsidize the implementation of new Centers for Patient Safety in health services.

Descriptors: Emergency Medical Services. Nursing. Patient Safety.

Objetivo: entender la implementación del Centro de Seguridad del Paciente en una Unidad de Atención de Urgencia desde la perspectiva de las enfermeras. Método: estudio de caso cualitativo, realizado en una unidad de atención de urgencia en una ciudad de la región del medio oeste de Minas Gerais, Brasil, con la participación de 12 enfermeras. Los datos se recogieron de septiembre a noviembre de 2019, a través de una entrevista guiada por un guión semiestructurado, la Técnica del Cómic y la observación. Para el análisis se adoptó el análisis de contenido. Resultados: surgieron dos categorías temáticas: Desafíos de la implementación del Núcleo de Seguridad del Paciente e Facilidades de la implementación del Núcleo de Seguridad del Paciente. Conclusión: se identificaron debilidades y desafios que repercuten en el proceso de aplicación del núcleo y, por consiguiente, en la atención al paciente, lo que puede servir para subvencionar la implantación de nuevos Núcleos para la Seguridad del Paciente en los servicios de salud.

Descriptores: Servicios Médicos de Emergencia. Enfermería. Seguridad del paciente.

\section{Introdução}

A Unidade de Pronto Atendimento (UPA) surgiu como uma das estratégias da Política Nacional de Atenção às Urgências para melhor organizar a assistência, articulando os serviços de saúde e definindo fluxos. É um componente fixo pré-hospitalar que realiza atendimentos a casos agudos em situação de urgência e emergência $^{(1)}$. Contudo, estudos demonstraram uma assistência ineficaz, comportamentos e práticas inadequados, além da falta de recursos materiais, humanos e financeiros ${ }^{(2-3)}$, fatores que impactam diretamente na segurança e no processo do trabalho.

Buscando minimizar os riscos provenientes dos serviços de saúde, as instituições têm implementado iniciativas voltadas para a segurança do paciente ${ }^{(4-5)}$. Pode-se destacar a implementação do Núcleo de Segurança do Paciente (NSP); um caminho que o Ministério da Saúde (MS) instituiu como obrigatório e que visa melhorias e mais segurança ao paciente ${ }^{(6-7)}$.

Esse núcleo deve realizar a prevenção e o controle de incidentes nos serviços de saúde, promover um ambiente assistencial seguro, estimular a criação e a manutenção de uma cultura de segurança, organizar estratégias e ações para minimizar os riscos. Para isso, o NSP trabalha com as seis metas internacionais de segurança, a saber: identificação correta dos pacientes, comunicação efetiva, segurança na administração dos medicamentos, cirurgia segura, redução de infecções e prevenção de danos ${ }^{(6)}$.

O enfermeiro muitas vezes é o profissional coordenador do NSP, e é considerado o responsável por implementar ações estratégicas de segurança, pois possui habilidades gerenciais e assistenciais para auxiliar nas soluções de problemas, identificação de falhas, melhoria de resultados e padronização da assistência. Além disso, consegue gerenciar os principais riscos que os pacientes sob seus cuidados estão expostos, sejam eles químicos, físicos, psíquicos, sociais, espirituais, assistenciais e institucionais ${ }^{(5,8)}$.

Assim, o enfermeiro possui papel crucial no manejo, implementação e continuidade do NSP, pois é capaz de viabilizar estratégias que efetive a cultura de segurança na instituição e, como consequência, diminuir as ocorrências de possíveis eventos adversos que possam comprometer a integridade do paciente, familiar e acompanhante ${ }^{(9-10)}$.

Estudo $^{(4)}$ aponta que o NSP, por meio de seus membros, deve desempenhar medidas de educação e divulgação das boas práticas para profissionais de saúde, pacientes e acompanhantes; entretanto, só a criação do núcleo pode ser incipiente. Os seus membros devem desenvolver ações preventivas e mecanismos que possibilitem uma melhor capacitação, planejamento e divulgação das ações estratégicas do NSP, buscando garantir um cuidado seguro aos pacientes. 
No contexto da UPA, o profissional enfermeiro já desenvolve papel fundamental, exercendo a liderança da equipe, com visão ampliada, realizando estratégias simples e efetivas para prevenir e reduzir os riscos durante a permanência do paciente na instituição, na tentativa de garantir a qualidade do cuidado ${ }^{(2,7)}$.

Tendo em vista as considerações apresentadas, necessário se faz aprofundar a compreensão da implantação do NSP na perspectiva de enfermeiros, haja vista sua relevância no processo de implantação. Nessa ótica, o enfermeiro precisa estar alinhado com a proposta do NSP, com visão positiva em seu contexto de trabalho. Tal percepção positiva influencia na maneira como ele executa suas atividades, o que pode interferir nas ações desenvolvidas no NSP. Contudo, as inúmeras demandas de trabalho, com elevado número de atendimento, excesso de atividades burocráticas, escassez de pessoal e de material e outras dificuldades estruturais podem afetar negativamente a percepção do enfermeiro sobre o NSP ${ }^{(3,8)}$. Indaga-se, pois: "Como ocorreu a implementação do NSP na UPA? Qual a percepção do enfermeiro acerca do NSP?"

Este estudo justifica-se, pois, a estratégia de implementação do NSP ainda é algo novo para as instituições de saúde, o que configura um processo que está em construção nos serviços de urgência, assim como a implantação de medidas de boas práticas nesses cenários ${ }^{(4)}$.

Pressupõe-se que a implementação do NSP na UPA pode trazer melhorias nos serviços assistenciais e gerenciais e, no que tange à percepção do enfermeiro, por ser o líder da equipe, espera-se uma visão ampliada desse profissional, utilizando as estratégias do NSP como base para tomada de decisões relacionadas à qualidade e à segurança da assistência.

O objetivo do presente estudo é compreender a implementação do NSP em uma UPA, na perspectiva dos enfermeiros.

\section{Método}

Trata-se de estudo de caso único qualitativo, que permite entender como e por que um evento ocorre ${ }^{(11)}$, bem como investigar o cotidiano e as experiências interpretadas e reinterpretadas pelos sujeitos ${ }^{(12)}$.

O estudo foi realizado em um município da Região Centro-Oeste de Minas Gerais, Brasil, denominado como Município Y. A cidade possui apenas uma UPA, que realiza atendimento da população local e de municípios da região.

As UPAs foram criadas para atendimento a situações de urgência e emergência, articulando esse atendimento nos níveis primário, secundário e terciário ${ }^{(2)}$. A unidade deste cenário de estudo funciona 24 horas, realiza classificação de risco, presta atendimento a pacientes acometidos por quadros agudos e agudizados, construindo fluxos de referência e contrarreferência com outras instituições da região, e realiza uma média de 450 atendimentos diários.

Os participantes do estudo foram 12 enfermeiros, escolhidos intencionalmente. Acredita-se que a implantação do NSP repercute no trabalho desses profissionais, e que estes são figuras centrais na implementação e consolidação das ações estratégicas do Núcleo. Os critérios de inclusão foram: possuir vínculo empregatício com a UPA, independentemente do tempo, executar ações e procedimentos de enfermagem e ter vivenciado o processo de implementação do NSP. Utilizou-se como critério de exclusão estar de férias ou de licença no momento da coleta. Todos os enfermeiros da UPA participaram da pesquisa e não houve perda amostral.

A coleta de dados foi realizada por meio de entrevistas guiadas por roteiro semiestruturado, pela Técnica do "Gibi" e a observação. A coleta ocorreu entre setembro e novembro de 2019 e foi realizada por uma das pesquisadoras, devidamente capacitada, para garantir os critérios de elegibilidade e credibilidade.

Antes do início da coleta de dados, realizou-se uma entrevista piloto com uma enfermeira que já havia trabalhado anteriormente na instituição, com o intuito de verificar a aplicabilidade do roteiro de entrevista e da Técnica do Gibi. Após a fase piloto não houve necessidade de alteração no instrumento de coleta de dados. A entrevista piloto não foi utilizada para fins de análise. 
As entrevistas foram previamente agendadas, audiogravadas e realizadas na unidade, em uma sala reservada, possibilitando a coleta individual de dados, sem interferência, preservando o sigilo e o anonimato dos informantes, com duração média de 10 minutos. Para garantir o anonimato dos participantes, adotou-se a letra $\mathrm{E}$ de Enfermeiro, seguida pelo número da ordem da entrevista, gerando E1, E2, E3...

O perfil dos participantes foi delineado mediante a realização de perguntas específicas feitas anteriormente à realização da entrevista, e diziam respeito à percepção do enfermeiro sobre a implementação do NSP, com o seguinte detalhamento: O que o NSP significa para você? Qual a contribuição do NSP para seu trabalho? Quais as facilidades e dificuldades encontradas no trabalho do NSP? Qual a participação do enfermeiro no NSP?

As entrevistas foram transcritas na íntegra, e enviadas aos participantes para garantir que haviam verbalizado o que estava transcrito, garantindo a legitimidade. Relata-se que não se obteve retorno de nenhum entrevistado.

Imediatamente após a entrevista, foi realizada a Técnica do Gibi, que consiste em uma estratégia de coleta de dados que lança mão de histórias em quadrinhos para instigar o participante a se expressar por meio de figuras do gibi sobre um determinado tema ${ }^{(13)}$. Assim, a Técnica do Gibi favoreceu a expressão da subjetividade dos enfermeiros sobre a implementação do NSP na UPA.

Foi escolhida a revista de gibi do tipo "Turma da Mônica" por representar ações do cotidiano e por ser reconhecida pelos participantes. Escolheu-se, aleatoriamente, a última edição da revista, número 53, publicada em setembro de 2019, mês que iniciou a coleta de dados. A aplicação dessa técnica já foi utilizada anteriormente no ensino, pesquisa e assistência de enfermagem, contribuindo para o processo de reflexão, sensibilidade e comunicação ${ }^{(13)}$.

O participante pode escolher qualquer figura de toda a extensão da revista, incluindo capa, contracapa e propaganda, que representasse a resposta da seguinte pergunta: Como ocorreu o processo de implementação do NSP na unidade? Ressalta-se que os depoimentos gravados e transcritos com a descrição das figuras foram submetidos à análise de conteúdo.

A observação foi realizada com a finalidade de captar informações relevantes sobre atitudes, situações e comentários, objetivando reduzir a distância entre o discurso e a prática dos enfermeiros. Os dados foram registrados em diário de campo, compondo as notas de observação e teve a duração de 6 horas.

Para a análise dos dados, utilizou-se a Análise de Conteúdo proposta por Bardin, buscando alcançar a interpretação mais profunda do fenômeno, além de ultrapassar o alcance meramente descritivo do conteúdo manifesto da mensagem ${ }^{(14)}$. Assim, a análise dos dados foi realizada em torno das fases cronológicas: pré-análise, exploração do material e tratamento dos resultados, inferência e interpretação à luz da literatura. Portanto, inicialmente foi realizada a leitura flutuante e exaustiva das questões das entrevistas, de forma a interagir com o texto e obter a compreensão sobre o que o participante buscava transmitir. Em seguida, procedeu-se a exploração do material colhido, tornando as informações cada vez mais claras e apropriadas ao propósito do estudo, e por fim, o tratamento dos resultados, no qual as pesquisadoras buscaram torná-los significativos e analisá-los à luz da literatura. Foram elaboradas duas categorias: "Desafios da implementação do Núcleo de Segurança do Paciente" e "Facilidades da implementação do Núcleo de Segurança do Paciente".

A pesquisa seguiu as normas da Resolução 466/2012 e 580/2018 do Conselho Nacional de Saúde (CNS) e foi aprovada pelo Comitê de Ética em Pesquisa (CEP) da Universidade do Estado de Minas Gerais (UEMG), Belo Horizonte, sob o Parecer n. 3.535.414. Ressalta-se que os entrevistados assinaram o Termo de Consentimento Livre e Esclarecido (TCLE).

O projeto e o artigo foram elaborados levando em consideração o Consolidated Criteria for Reporting Qualitative Research (COREQ).

\section{Resultados}

Participaram 12 enfermeiros, 4 homens e 8 mulheres. Em relação à faixa etária, 25\% $(n=3)$ 
dos participantes tinham entre 40 e 49 anos e $75 \%$ $(n=9)$ entre 30 e 35 anos; quanto ao estado civil, $33 \%(n=4)$ solteiros, $8 \%(n=1)$ viúvos e $58 \%(n=7)$ casados. Todos cumpriam a carga horária de 30 horas semanais e trabalhavam em regime de dedicação exclusiva, não possuindo outro vínculo.

Dentre os entrevistados, 75\% (n=9) atuavam como supervisores de setores como ambulatório, cirurgia, ortopedia, pediatria, enfermaria e emergência e 25\% (n=3) na supervisão da classificação de risco. Com relação ao contrato de trabalho, 75\% ( $n=9)$ dos profissionais eram celetistas e 25\% (n=3) concursados. Apenas 17\% $(n=2)$ apresentavam especialização na área de segurança do paciente, e 42\% $(n=5)$ possuíam título de mestre.
Por meio da observação, foram levantados dados sobre a formação do NSP na UPA. O NSP foi fundado em julho de 2018, constituído por cinco enfermeiros, dois técnicos de enfermagem, um médico clínico geral, uma farmacêutica, uma supervisora da hotelaria e copa, e um atendente de portaria. Ressalta-se que os cargos de coordenação e tesouraria, no núcleo, eram ocupados por enfermeiros.

Da análise resultaram as seguintes categorias: Desafios da implementação do Núcleo de Segurança do Paciente e Facilidades da implementação do Núcleo de Segurança do Paciente. Para facilitar a compreensão, as categorias e subcategorias foram sintetizadas no Quadro 1.

Quadro 1 - Categorias e subcategorias da implementação do Núcleo de Segurança do Paciente em uma Unidade de Pronto Atendimento, na perspectiva dos enfermeiros

\begin{tabular}{|c|c|}
\hline Categorias & Subcategorias \\
\hline \multirow[t]{4}{*}{$\begin{array}{l}\text { Desafios da implementação do Núcleo de } \\
\text { Segurança do Paciente }\end{array}$} & $\begin{array}{l}\text { Falta de interesse e de apoio financeiro por parte } \\
\text { da gestão do Município }\end{array}$ \\
\hline & Superlotação da Unidade de Pronto Atendimento \\
\hline & Sobrecarga de trabalho da equipe \\
\hline & $\begin{array}{l}\text { Comunicação ineficaz com a equipe } \\
\text { multidisciplinar }\end{array}$ \\
\hline \multirow[t]{5}{*}{$\begin{array}{l}\text { Facilidades da implementação do Núcleo de } \\
\text { Segurança do Paciente }\end{array}$} & $\begin{array}{l}\text { Conhecimento prévio dos profissionais sobre } \\
\text { segurança do paciente }\end{array}$ \\
\hline & Capacitação da equipe \\
\hline & Motivação da equipe multidisciplinar \\
\hline & $\begin{array}{l}\text { Composição do Núcleo de Segurança do } \\
\text { Paciente por uma equipe multidisciplinar }\end{array}$ \\
\hline & $\begin{array}{l}\text { Vinculação com a Semana Interna de Prevenção } \\
\text { de Acidentes de Trabalho }\end{array}$ \\
\hline
\end{tabular}

Fonte: Elaboração própria.

\section{Desafios da implementação do Núcleo de Segurança do Paciente}

Nesta categoria são abordados os desafios identificados na implementação do NSP.

Os enfermeiros relataram a falta de envolvimento e apoio da gestão para implementar o NSP, conforme ilustrado:

Se houve uma grande dificuldade nessa implementação, sem dúvidas foi a falta de apoio e também de interesse direto da gestão, eles não têm interesse [...] segurança do paciente não enche os olhos de secretário de saúde, até mesmo porque comprar uma ambulância ou pintar a unidade para a população faz mais vista. Cultura de segurança é algo que não é percebido e pouco valorizado pela gestão daqui. (E8).

Mas existe uma questão política envolvida na saúde do Município $Y$, inclusive na UPA do Município Y tudo que pedimos e solicitamos foi barrado diretamente. Oficializando um não funcionamento por falta de apoio gestorial. É triste! (E10).

Por meio da Técnica do Gibi foi ressaltada a ausência dos gestores nas discussões sobre o NSP, o que imprime um desinteresse no que tange à importância institucional conferida à segurança, como consta na Figura 1. 
Figura 1 - Figura originada da Técnica do Gibi

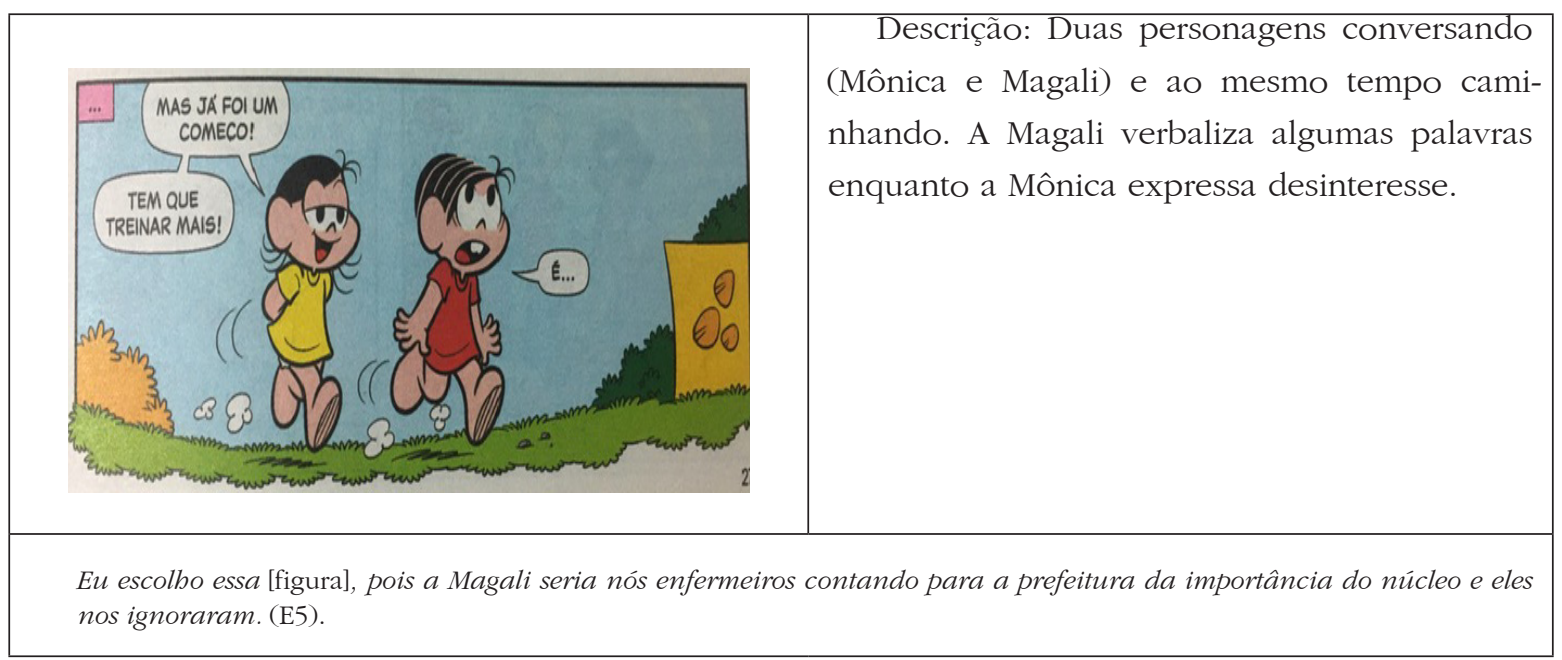

Fonte: Sousa $\mathrm{M}^{(15: 27)}$.

Os enfermeiros entendem que é primordial o apoio da gestão para promover políticas institucionais e desenvolver ações que envolvam a segurança do paciente. Também relatam a falta de apoio financeiro da alta gestão do município:

Com toda certeza, o que nos barrou diretamente foi à falta de apoio financeiro, de recursos mesmos, para poder investir em segurança e, também, na administração. Digo, prefeitura mesmo e o gestor geral nos desanimaram bastante. (E5).

Bom, maior dificuldade foi financeira né, porque a gente não tinha muito auxílio da parte da gestão mesmo, porque nós precisamos de melhorias de materiais! (E9).

Os participantes relataram que a superlotação de pacientes na unidade e a consequente sobrecarga de trabalho foram barreiras da implantação do NSP:

Eu acho que umas das dificuldades também seja o número da equipe. São poucos técnicos para tanta gente. No PS1 [Pronto Socorro 1] que é o setor mais cheio, as vezes fica com 50 pacientes internados para quatro funcionários e ainda precisamos atender porta e medicação externa. É um caos! (E1).

Às vezes um técnico pela manhã pega um corredor com 17 pacientes e precisam prestar todos os cuidados, e tudo misturado: bomem, mulher, idosos, acamados, de sonda, fralda, curativo para ser feito. Todos distribuídos em macas no corredor, os técnicos pulam igual pipoca as 12 horas para dar conta de tudo. Fazer o básico, como, por exemplo, dupla checagem, essas coisas pedidas pelo núcleo ficam quase impossivel de ser feita! (E12).

Além dos relatos, foi observada a ocupação da totalidade dos leitos na UPA, pacientes nos corredores, alta tensão da equipe assistencial durante a execução de procedimentos técnicos, pressão da população e do serviço para realizar novos atendimentos, e tempo de espera acima de uma hora. A sobrecarga da equipe dificulta a assistência segura, conforme preconiza o NSP, levando a uma desatenção nas práticas de saúde e contribuindo para o surgimento de erros e incidentes relacionados ao cuidado.

O entrevistado E7 na Técnica do Gibi relatou que são diversas as atribuições da equipe e que isso sobrecarrega o funcionário. O depoimento trata o Núcleo como algo não consolidado, não funcionante na UPA, o que repercute no trabalho da equipe no que tange à segurança do paciente, como apresentado na Figura 2. 
Figura 2 - Figura originada da Técnica do Gibi

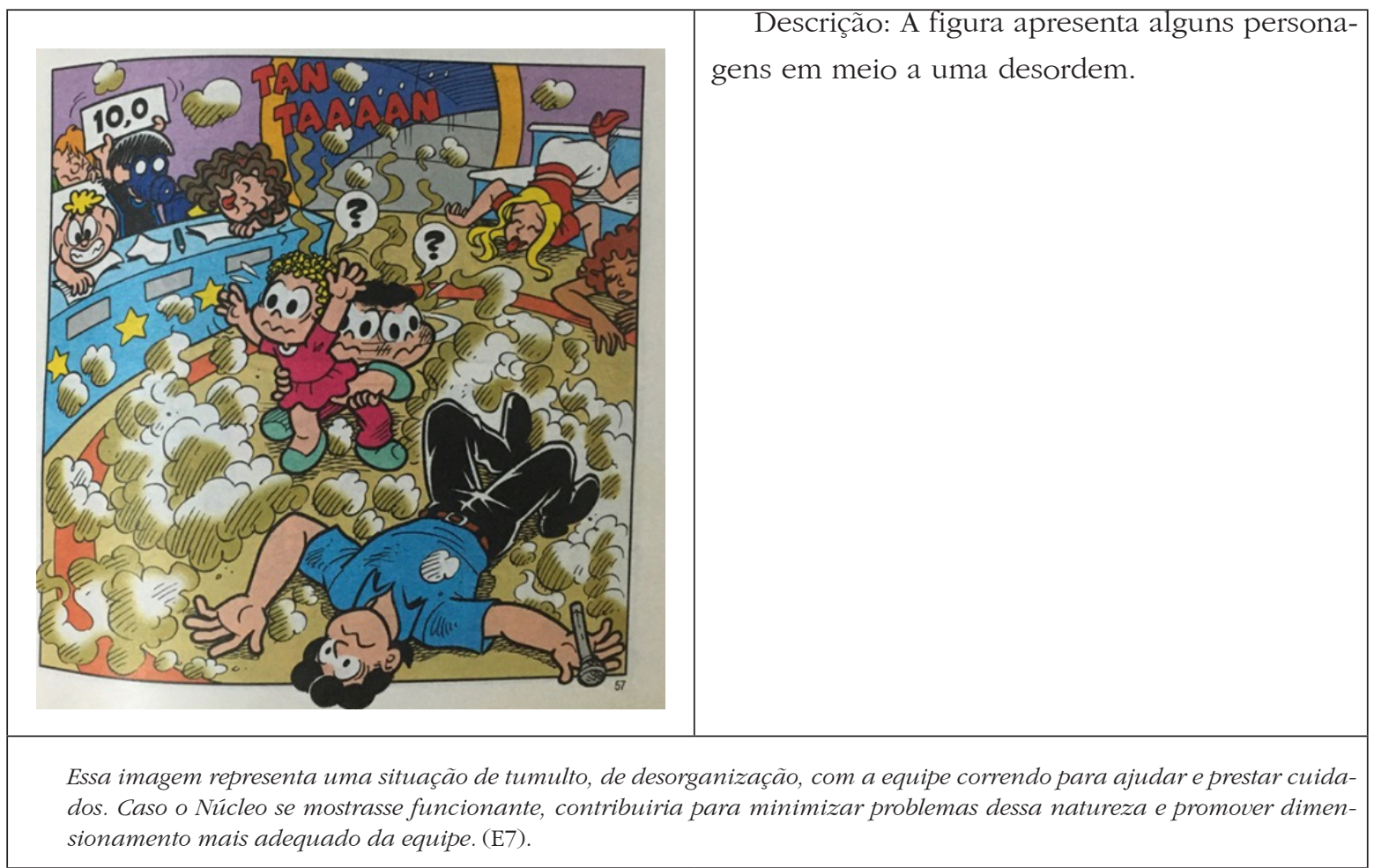

Fonte: Sousa $\mathrm{M}^{(15: 57)}$.

Os depoimentos apontam o problema da comunicação ineficaz entre a equipe, escrita ou verbal, impedindo a implementação e a continuidade do NSP:

[...] a gente tem uma comunicação muito falha e o processo de comunicação falho seja ele de registros, verbais e as comunicaçôes profissionais da efetividade das informações da continuidade, é algo muito falho [...] a comunicação, ela depende muito do subjetivo do profissional, do entendimento que aquele profissional tem sobre a importância da comunicação, da informação assertiva, da informação correta, né? (E2).

Acho que se a gestão comunicasse mais com o resto, mais com a equipe, né [...] o processo teria acontecido mais rápido, acontecido efetivamente. (E4).

Esses desafios se constituem no trabalho da enfermagem, de outros profissionais de saúde e na implementação das ações do NSP; assim, é necessário serem repensados para favorecer as práticas seguras.

\section{Facilidades da implementação do Núcleo de Segurança do Paciente}

Nesta categoria serão apresentadas as facilidades encontradas no processo de implementação do NSP. Inicialmente podemos citar que o conhecimento prévio da temática segurança do paciente foi levantado como fator importante para implementação do NSP, como exposto por E3 e E5:

O que me ajudou muito como vice-presidente do núcleo foi ainda está estudando, eu tive mais facilidade, até mesmo tinha mais conhecimento, graças ao mestrado a gente quando se mantém na academia está mais atualizado [...] também mais aberto a novas mudanças. (E3).

Por um lado mais otimista, nós temos enfermeiros com facilidade na criação do núcleo, por já estarem inseridos no mestrado ou recém-formados, assim, tiveram uma grande contribuição da graduação no que se refere a formação do núcleo. Essas foram algumas poucas das facilidades que nós tivemos. (E5).

O conhecimento prévio dos profissionais sobre a temática e a importância do NSP na instituição são fatores de promoção do debate no serviço, auxiliando no desenvolvimento da cultura de segurança, com foco na redução de riscos e de erros. Os enfermeiros acrescentam que a atualização da equipe faz com que esta se sinta mais segura, estimulada e interessada na busca de melhorias.

A capacitação é apontada como facilitadora, conforme ilustrado na Figura 3. 
Figura 3 - Figura originada da Técnica do Gibi

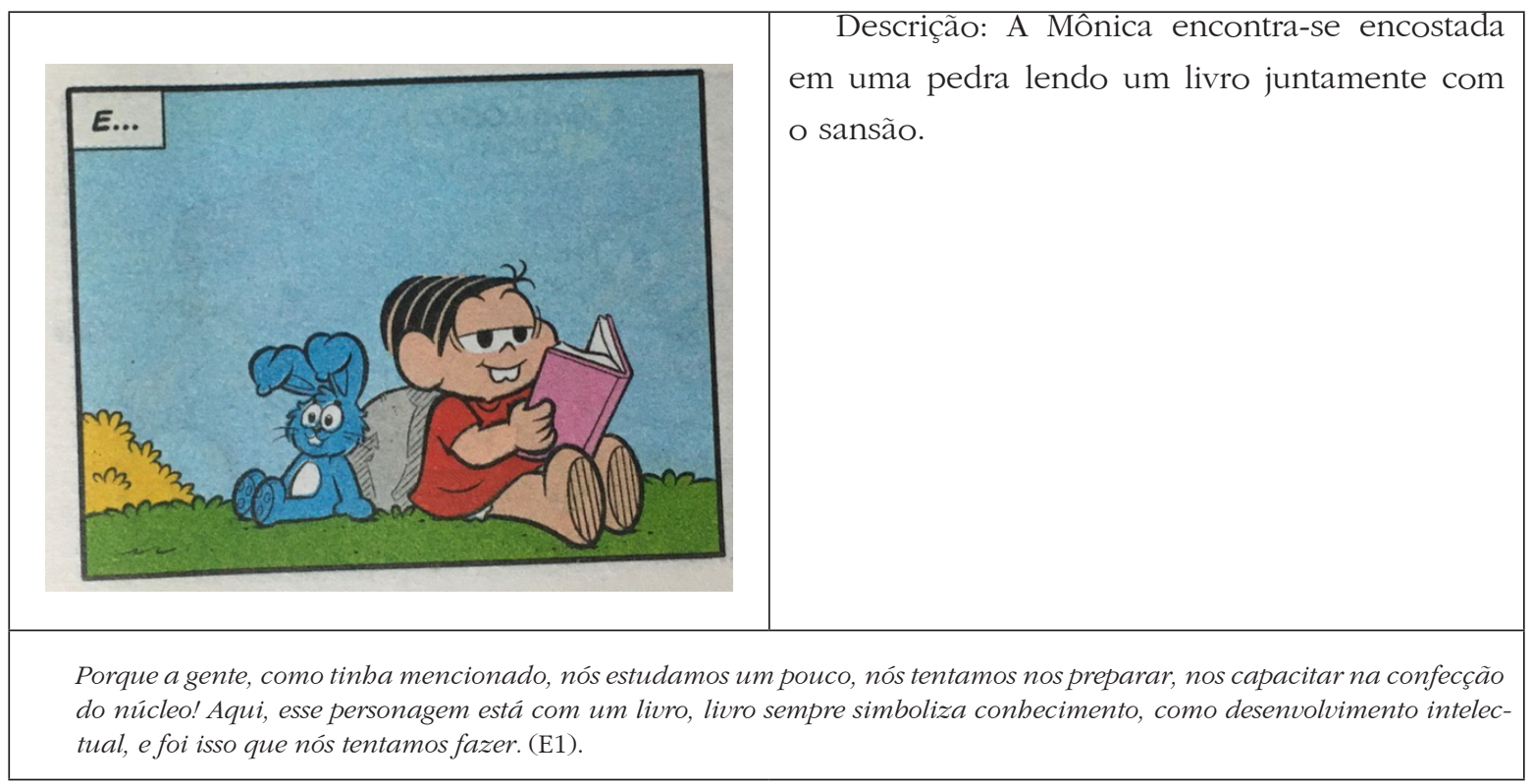

Fonte: Sousa $\mathrm{M}^{(15: 9)}$.

A capacitação é vista como o meio para incentivar e compartilhar conhecimentos, além de possibilitar a qualificação e a revisão de conteúdo, como acrescentado por E6:

Apesar de alguns enfermeiros terem mais tempo de formado ainda são bem interessados em continuar se especializando, isso parte de cada um, mas a equipe daqui tem isso na essência [...] graças a Deus! (E6).

Adiciona-se as contribuições da ideia da responsabilidade coletiva, e assim, entende-se que há cooperação, união, respeito e motivação. O embasamento teórico e o estudo científico facilitam a implantação do NSP, pois deixa o profissional aberto às novas mudanças, normas e compreensão da importância de novas ações, como relatado nos depoimentos:

[...] é a contribuição da equipe, participação da equipe em manter a segurança do paciente, trabalhar de forma correta e coerente. (E7)

[...] com certeza o apoio e conbecimento da equipe são muito importantes, acho que a única coisa que nos facilitou foi isso mesmo e só. (E11).

A motivação foi relatada como facilitadora. A despeito dos desafios apresentados, os enfermeiros se sentiam motivados a melhorar o serviço, sendo esse um elemento crucial para o incentivo e potencialização da cultura de segurança do paciente na UPA. Os enfermeiros reconhecem que quando os profissionais estão motivados e estimulados, realizam movimentos em prol de mudanças, de melhoria no ambiente de trabalho, com incentivo à cultura de segurança.

Outro facilitador apontado pelos enfermeiros foi a definição de uma equipe multidisciplinar para compor, como membros, o NSP, conforme depoimento:

[...] a integração de diversos perfis profissionais na composição do núcleo foi um ponto facilitador, porque ai a gente levou profissionais que tem visões diferentes e que tinham uma facilidade de adequação dentro da sua rotina. (E9).

O trabalho multiprofissional, os conhecimentos que contribuíam para o trabalho em equipe em um clima harmônico, de corresponsabilidades e respeito, são elementos importantes para a implementação do NSP. Ressalta-se também a necessidade de compreensão das especificações de cada paciente, de forma global, e a atuações de diversas profissões.

Foi observado que a inserção da Semana Interna de Prevenção de Acidentes de Trabalho (SIPAT) foi um facilitador na implementação do NSP, conforme enfatizado pela fala de E9:

A ajuda que a gente teve da SIPAT que é uma instituição que tem na unidade de certa forma teve uma atividade maior né, junto com a segurança do paciente em si. (E9).

Pode-se dizer que o SIPAT e o NSP possuem atividades semelhantes e seus membros visam 
conscientizar os profissionais em relação à prevenção de doenças ocupacionais, acidentes de trabalho, estimular a promoção da saúde e proteção da integridade física. Ainda, buscam promover treinamentos de segurança do paciente e do profissional.

\section{Discussão}

Para construir e implementar o NSP é necessário o envolvimento da gestão, da UPA e do município, visto que requer adequações estruturais, materiais e estratégicas para garantir a segurança do paciente e do funcionário ${ }^{(7)}$.

Estudo realizado com enfermeiros em quatro hospitais universitários públicos do estado do Paraná apontou que a falha no apoio por parte da gestão é um fator que dificulta a implementação de planos de segurança ${ }^{(5)}$. Os gestores possuem poder de decisão para nomear membros para compor o NSP, conferindo-os autoridade e responsabilidade para executarem a promoção de um cuidado seguro ${ }^{(16)}$.

A equipe espera ser informada pela gerência sobre os problemas de segurança da unidade, o que, quando ocorre, gera sentimentos positivos na equipe, como de apoio e incentivo à possibilidade de expor opiniões e impressões em relação às decisões. Situações contrárias acarretam insegurança na equipe ${ }^{(17)}$.

Um estudo apontou que o apoio da alta direção e o engajamento das lideranças foram fundamentais para a implementação e o desenvolvimento do NSP, analisando as informações e auxiliando na implantação de melhorias ${ }^{(7)}$. Os gestores devem estar cientes dos benefícios do NSP e fornecer evidências do seu comprometimento, além de buscar melhorar a satisfação do paciente, definir as competências dos profissionais envolvidos e os processos ${ }^{(16)}$.

Para garantir a segurança do paciente é necessária a criação de políticas, rotinas e normas, devendo haver investimentos em infraestrutura, equipamentos e materiais. Esses fatores são potenciais dispositivos de mudanças na assistência e, quando ausentes, tornam as atividades lentas ${ }^{(3)}$.
Em relação à superlotação de pacientes e a consequente sobrecarga de trabalho, pode-se dizer que o grande fluxo de pacientes decorre frequentemente da demanda por atendimentos que não se caracterizam como urgência. Nessa direção, estudo(2) desenvolvido em UPAs de um município de Santa Catarina reforça que a superlotação ocorre devido a falha do sistema de saúde em absorver a demanda da atenção primária, levando os usuários a buscarem atendimentos viáveis, independente da sua complexidade. Assim, a população reconhece a UPA como um local de acesso e de resolutividade. O atendimento torna-se demorado gerando estresse no paciente e na equipe.

Estudo alemão concluiu que o aumento da carga de trabalho dos médicos e enfermeiros está relacionado ao aumento da complexidade de doenças e à necessidade de desenvolver habilidades devido à inovação tecnológica. Aponta, ainda, que a sobrecarga gera tensão e estresse nos profissionais e que para garantir a segurança do paciente é necessário ter uma liderança capaz de gerenciar e contrabalancear a demanda do trabalho, ressaltando que ouvir o profissional é fundamental nesse processo ${ }^{(18)}$.

A sobrecarga de trabalho está relacionada à desproporção entre o número de profissionais de enfermagem e de pacientes atendidos, relatada como fator de risco para ambos. Esses desafios podem contribuir para o surgimento de erros e incidentes relacionados ao cuidado, constituindo-se como um desafio para a segurança do paciente ${ }^{(9)}$.

A falta de comunicação efetiva representa um dos principais desafios à identificação de incidentes e eventos adversos. O trabalho em equipe requer comunicação entre todos os envolvidos para alcançar os resultados almejados com sucesso, eficiência e menos erros. Assim, devem ser identificadas barreiras que impedem a comunicação eficaz e que inviabilizam a cooperação entre a equipe ${ }^{(19)}$. A má comunicação é um problema persistente nos cuidados de saúde, devido a algumas abordagens teóricas e definições restritas que os profissionais utilizam. Sugere-se que sejam construídos relacionamentos 
10

Implementação de Núcleo de Segurança do Paciente em Unidade de Pronto Atendimento: perspectivas dos enfermeiros

de confiança entre os atores, além de expandir a definição do valor da comunicação ${ }^{(20)}$.

A comunicação deve ser universal, dialogada e registrada, além de haver uma escuta que favoreça o aprimoramento de práticas seguras e promoção da redução de ocorrência de erros ${ }^{(19)}$. Essa afirmação corrobora estudo ${ }^{(21)}$ que aborda que a comunicação deve promover a troca de ideias e experiências para gerar mudanças de comportamento, discutir e ensinar assuntos novos, o que se aplica à segurança do paciente.

A segurança do paciente precisa ser trabalhada nos cursos de graduação e de pós-graduação, bem como no desenvolvimento contínuo do profissional. Estudo realizado em quatro hospitais palestinos revela que embora os enfermeiros possuam deficiência na temática segurança do paciente, ainda conseguem ter atitudes positivas em relação ao cuidado. Torna-se, pois, fundamental, que as discussões iniciem-se na graduação, para que o profissional tenha uma formação clínica embasada cientificamente em práticas seguras ${ }^{(22)}$.

Nesse contexto, a segurança do paciente deve ser uma ciência básica para a educação profissional, expondo conceitos, atitudes e habilidades para a prática da segurança e promoção de melhorias no cuidado. Inclui ainda, cinco competências, quais sejam: cuidado centrado no paciente, capacidade de trabalhar em equipe, práticas baseadas em evidência, melhoria da qualidade e utilização da informática. Essa mudança já está sendo incorporada em algumas escolas dos Estados Unidos, Reino Unido e países de língua alemã ${ }^{(23)}$.

O NSP possui como atividade a difusão e a capacitação periódica dos profissionais. Desse modo, o serviço precisa desenvolver, implementar e acompanhar programas de capacitação voltados para a segurança do paciente ${ }^{(6)}$.

Quando os profissionais se capacitam criam responsabilidade coletiva. Sugere-se que a capacitação seja realizada por meio de práticas reflexivas e rodas de conversa, para estimular o bom relacionamento e a cooperação na equipe. Desse modo, haverá apoio para a incorporação da cultura de segurança de modo enraizado e sistêmico na instituição ${ }^{(24)}$.

A participação dos profissionais em ações que modificam o processo de trabalho gera percepções positivas pela oportunidade de vivenciar novas experiências e de ampliar conhecimento, além da satisfação em proporcionar segurança ao paciente, e saber que isso gera benefícios e traz satisfação e credibilidade ${ }^{(5)}$. A implementação do NSP no serviço não pode ser vista somente como mudança em recursos, estrutura e equipamentos, mas inciativas humanas. Além disso, as pessoas e os grupos precisam ser estimulados por suas lideranças.

Para o desenvolvimento da cultura de segurança, autores ${ }^{(16)}$ relataram que o NSP requer a composição, preferencialmente por membros da organização que tenham noção de processos de trabalho e possuam perfil de liderança. É ideal que tenha conhecimento sobre conceitos de melhoria da qualidade e temáticas, como controle de infeção, gerenciamento de risco, epidemiologia, microbiologia, qualidade, farmácia e engenharia clínica e segurança do paciente.

O trabalho em equipe deve ser realizado com base no diálogo, feedback e laços de confiança. Além disso, deve promover pensamentos críticos sobre ações e atitudes e alternativas para modificar e transformar o erro ${ }^{(25)}$.

O trabalho em equipe comporta o compartilhamento de histórias, conhecimento, insights, proatividade, encontro com outros membros, preparo para resolver uma quebra de informação e criação de estratégias para prevenção de falhas. As experiências relatadas por esse grupo permitem o aprendizado e podem ser replicadas e melhoradas, com vistas a segurança do paciente ${ }^{(26)}$.

O NSP tem como atividade identificar e conhecer os processos de cuidado que podem ter pontos críticos e gerar riscos. Dessa forma, deve estabelecer barreiras capazes de prevenir incidentes e mostrá-las aos seus profissionais ${ }^{(6)}$.

Cabe salientar que esse processo de implementação do NSP indica a necessidade de investimentos, iniciativas de educação e capacitação, sensibilização e envolvimento da alta gestão, 
profissionais e pacientes com intuito de oferecer atenção segura e de qualidade.

A principal limitação da pesquisa foi a redução do número de enfermeiros no serviço, devido à diminuição nas contratações. Esse fato fez com que fosse empregado o critério de totalidade para alcançar o objetivo proposto.

\section{Conclusão}

O estudo identificou que existem desafios na implementação do NSP na perspectiva dos enfermeiros, que destacaram a falta de interesse e de apoio financeiro por parte da gestão do município; a superlotação da UPA; a sobrecarga de trabalho e a comunicação ineficaz. Em contrapartida, foram identificadas as facilidades na implementação do NSP: o conhecimento prévio sobre segurança do paciente, a capacitação dos profissionais, a motivação da equipe, a composição do NSP por uma equipe multidisciplinar e a vinculação com a SIPAT.

Este estudo traz como contribuições para a enfermagem, a compreensão de que a implantação do NSP ainda é algo desafiador para os serviços de saúde, mas o enfermeiro possui competências e habilidades que auxiliam na implementação do NSP, contribuindo com a cultura de segurança organizacional e intermediando ações gerenciais entre a gestão da unidade e a equipe multiprofissional.

Além disso, este artigo visa encorajar os serviços de saúde a trilhar o caminho da segurança do paciente com o auxílio da enfermagem, proporcionando uma assistência mais segura para a instituição, o paciente e os profissionais. É necessário criar estratégias que auxiliem na redução dos desafios encontrados, pois o NSP é essencial para o avanço da qualidade dos atendimentos, e, consequentemente, reduz os riscos e a vulnerabilidade do paciente.

Destaca-se a necessidade de outros estudos sobre as estratégias que podem ser utilizadas e que consigam mudar as práticas assistenciais e as condições estruturais, bem como estudos sobre a construção de uma cultura de segurança do paciente.
É importante evidenciar que o objetivo deste estudo não foi representar os profissionais, mas sim, compreender a implementação do NSP em sua perspectiva. Logo, o estudo contribuiu com discussões do tema na unidade, bem como poderá ser utilizado para nortear as equipes quanto à implementação do NSP nos demais serviços que tenham interesse e deu visibilidade ao tema de segurança.

\section{Agradecimentos:}

$$
\text { CAPES, FAPEMIG, CNPq, NUPAE. }
$$

\section{Colaborações:}

1 - concepção, projeto, análise e interpretação dos dados: Simone Graziele Silva Cunha, Gabriela Santos Clemence e Larissa Franciele Simões Almeida;

2 - redação do artigo e revisão crítica relevante do conteúdo intelectual: Simone Graziele Silva Cunha, Gabriela Santos Clemence, Larissa Franciele Simões Almeida, Andréia Guerra Siman e Maria José Menezes Brito;

3 - aprovação final da versão a ser publicada: Simone Graziele Silva Cunha, Andréia Guerra Siman e Maria José Menezes Brito.

\section{Referências}

1. O’Dwyer G, Konder MT, Reciputti LP, Lopes MGM, Agostinho DF, Alves GF. O processo de implantação das unidades de pronto atendimento no Brasil. Rev Saúde Pública. 2017;51:125. DOI: https://doi. org/10.11606/S1518-8787.2017051000072

2. Oliveira SN, Ramos BJ, Piazza M, Prado ML, Reibnitz SK, Souza AC. Unidade de Pronto Atendimento-UPA 24h: percepção da enfermagem. Texto contexto - enferm. 2015;24(1):238-44. DOI: https://doi.org/10.1590/0104-07072015003390011

3. Cavalcante EFO, Pereira IRBO, Leite MJVF, Santos AMD, Cavalcante CAA. Implementação dos núcleos de segurança do paciente e as infecções relacionadas à assistência à saúde. Rev Gaúcha Enferm. 2019; 40(esp):e20180306. DOI: https://doi. org/10.1590/1983- 1447.2019.20180306

4. Paixão DPSS, Batista J, Maziero ECS, Alpendre FT, Amaya MR, Cruz EDA. Adesão aos protocolos 
de segurança do paciente em unidades de pronto atendimento. Rev Bras Enferm. 2018;71(Suppl 1):622-9. DOI: http://dx.doi. org/10.1590/0034-7167-2017-0504

5. Reis GAX, Oliveira JLC, Ferreira AMD, Vituri DW, Marcon SS, Matsuda LM. Dificuldades para implantar estratégias de segurança do paciente: perspectivas de enfermeiros gestores. Rev Gaúcha Enferm. 2019;40(esp):e20180366. DOI: https://doi. org/10.1590/1983-1447.2019.20180366

6. Brasil. Agência Nacional de Vigilância Sanitária. Implantação do Núcleo de Segurança do Paciente em Serviços de Saúde [Internet]. Brasília (DF); 2016. (Série: Segurança do Paciente e Qualidade em Serviços de Saúde) [cited 2020 Mar 23]. Available from: http:// portal.anvisa.gov.br/documents/33852/3507912/ Caderno+6+-+Implanta $\% \mathrm{C} 3 \% \mathrm{~A} 7 \% \mathrm{C} 3 \% \mathrm{~A} 3 \mathrm{O}+\mathrm{do}+$ $\mathrm{N} \% \mathrm{C} 3 \%$ BAcleo+de+Seguran\%C3\%A7a+do+Pac iente+em+Servi\%C3\%A7os+de+Sa\%C3\%BAde/ cb237a40-ffd1-401f-b7fd-7371e495755c

7. Prates CG, Magalhães AMM, Balen MA, Moura GMSS. Núcleo de segurança do paciente: caminho das pedras em um hospital geral. Rev Gaúcha Enferm. 2019;40(esp):e20180150. DOI: https://doi.org/10.1590/1983- 1447.2019.20180150

8. Tondo JCA, Guirardello EB. Percepção dos profissionais de enfermagem sobre a cultura de segurança do paciente. Rev Bras Enferm. 2017;70(6):1355-60. DOI: http://dx.doi. org/10.1590/0034-7167-2016-0010

9. Siqueira CL, Silva CC, Teles JKN, Feldman LB. Gerenciamento de risco: percepção de enfermeiros em dois hospitais do Sul de Minas Gerais, Brasil. Rev Min Enferm. 2015;19(4):919-26. DOI: http:// www.dx.doi.org/10.5935/1415-2762.20150071

10. Carthon JMB, Davis L, Dierkes A, Hatfield L, Hedgeland T, Holland S, et al. Association of Nurse Engagement and Nurse Staffing on Patient Safety. J Nurs Care Qual. 2019;34(1):40-6. DOI: 10.1097/NCQ.0000000000000334

11. Yin RK. Estudo de Caso. Planejamento e Métodos. 5a ed. São Paulo: Bookman; 2015.

12. Minayo MCS. Teoria método e criatividade. 21a ed. Petrópolis: Vozes; 2015.

13. Brito MJM, Caram CS, Moreira DA, Rezende LC, Cardoso CML, Caçador BS. Técnica do Gibi como recurso metodológico aplicado na Enfermagem.
Rev baiana enferm. 2019;33:e29895. DOI: 10.18471/ rbe.v33.29895

14. Bardin L. Análise de conteúdo. São Paulo: Edições 70; 2011.

15. Sousa M. Produções. Revista da Turma da Mônica. 2019. n. 53.

16. Silva ACMR, Loures PV, Paula KX, Santos NAR, Perígolo R. A importância do núcleo de segurança do paciente: um guia para implantação em hospitais. Rev Educ Meio Amb Saú [Internet]. 2017 [cited 2020 Mar 23];7(1):87-109. Available from: file://C:/ Users/Simone\%20Cunha/Downloads/134-3671-PB.pdf

17. Nacioglu A. As a critical behavior to improve quality and patient safety in health care: speaking up! Saf Health. 2016;2(10):1-25. DOI: 10.1186/ s40886-016-0021-x

18. Sturm H, Rieger MA, Martus P, Ueding E, Wagner A, Holderried $M$, et al. Do perceived working conditions and patient safety culture correlate with objective workload and patient outcomes: A cross-sectional explorative study from a German university hospital. PLoS ONE. 2019;14(1):e0209487. DOI: 10.1371/journal.pone. 0209487

19. Gluyas H. Effective communication and teamwork promotes patient safety. Nurs Stand. 2015;29(49):50-7. DOI: 10.7748/ns.29.49.50.e10042

20. Manojlovich M, Hofer TPMD, Krein SL. Advancing patient safety through the clinical application of a framework focused on communication. J Patient Safety. 2018 Oct 31. DOI: 10.1097/ PTS.0000000000000547

21. Massoco ECP, Melleiro MM. Comunicação e segurança do paciente: percepção dos profissionais de enfermagem de um hospital de ensino. Rev Min Enferm. 2015;19(2):187-91. DOI: http://www. dx.doi.org/10.5935/1415-2762.20150034

22. Abu-El-Noor NI, Abu-El-Noor MK, Abuowda YZ, Alfaqawi M, Bottcher B. Patient safety culture among nurses working in Palestinian governmental hospital: a pathway to a new policy. BMC Health Serv Res. 2019;19:550 . DOI: 10.1186/ s12913-019-4374-9

23. Wu AW, Busch IM. Patient safety: a new basic Science for professional education. GMS J Med Educ. 2019;36(2):Doc21. DOI: 10.3205/zma001229 
Simone Graziele Silva Cunha, Gabriela Santos Clemence, Larissa Franciele Simões Almeida, Andréia Guerra Siman, Maria José Menezes Brito

24. Wegner W, Silva SC, Kantorski KJC, Predebon CM, Sanches MO, Pedro ENR. Educação para cultura da segurança do paciente: implicações para a formação profissional. Esc Anna Nery. 2016;20(3):e20160068. DOI: $10.5935 / 1414-8145.20160068$

25. Minuzzi AP, Salum NC, Locks MOH, Amante LN, Matos E. Contribuições da equipe de saúde visando à promoção da segurança do paciente no cuidado intensivo. Esc Anna Nery. 2016;20(1):121-9. DOI: $10.5935 / 1414-8145.20160017$
26. Zipperer L. "Humanness"- A crucial component of Knowledge sharing for patient safety. J Pat Saf Risk Manage. 2019;24(2):55-6. DOI: $10.1177 / 2516043519826751$

Recebido: 4 de abril de 2020 Aprovado: 30 de maio de 2020 Publicado: 6 de julho de 2020

A Revista Baiana de Enfermagem utiliza a Licença Creative Commons - Atribuição-NãoComercial 4.0 Internacional. https://creativecommons.org/licenses/by-nc/4.0/ Este artigo é de acesso aberto distribuído sob os termos da Licença Creative Commons (CC BY-NC). Esta licença permite que outros remixem, adaptem e criem a partir do seu trabalho para fins não comerciais. Embora os novos trabalhos tenham de lhe atribuir o devido crédito e não possam ser usados para fins comerciais, os usuários não têm de licenciar esses trabalhos derivados sob os mesmos termos. 